\title{
Evaluation of Actual Timetables and Utilization Levels of West Midlands Metro Using Event-Based Simulations
}

\author{
Praful Potti $^{1} \cdot$ Marin $^{\text {Marinov }^{1}}{ }^{(\mathbb{C})}$
}

Received: 24 June 2019/Revised: 30 November 2019/Accepted: 3 December 2019/Published online: 8 February 2020

(C) The Author(s) 2020

\begin{abstract}
The performance of the West Midlands Metro in the United Kingdom is analyzed in the present study by evaluating the existing timetables of the metro system. Using SIMUL8 computer software, a discrete event-based simulation prototype modeling the metro system is developed and implemented. The model adequately describes the performance of the West Midlands Metro system. By running simulations, the overall utilization level of the metro system is calculated. The results of the simulation model indicate that the metro system is being underutilized. The low utilization rates indicate a potential for the introduction of new services capable of exploiting the existing infrastructure and improving the utilization levels of the existing metro system; For example, the potential of using the current metro system for urban freight transport could be a new service of interest and provide scope for further research.
\end{abstract}

Keywords Metro $\cdot$ Rail - Timetable $\cdot$ Metro system performance - Utilization levels - Simulation - Modeling · SIMUL8 - Urban freight

\section{Introduction}

\subsection{Motivation}

As part of the long-term transport strategy of the West Midlands Combined Authority (WMCA) in the United

Marin Marinov

m.marinov@aston.ac.uk

1 Aston University, Birmingham, UK

Communicated by Waressara Weerawat.
Kingdom, the Midland Metro network is scheduled to triple in size over the next decade, with passenger numbers expected to grow over 30 million [1]. Metro service organizations invest heavily in the purchase of new vehicles, and often the trade between utilization levels and capital costs does not match. To achieve this balance, the prospective opportunities of exploiting the existing metro network is essential. This can eventually contribute to improving the utilization of the system. To carry out further research on the Midlands Metro system, it is important to study and understand the actual performance of the metro system. For that, evaluating the timetables and estimating the utilization levels of the system provide an opportunity to identify potential services that can be potentially introduced into the system later. This situation was the reason for assessing West Midlands Metro's schedules by developing a simulation model.

\subsection{Objectives}

The three key aims of the study are: first, to analyze the current timetable of West Midlands Metro system in the United Kingdom; Second, to generate the average waiting time and working time of each station in the existing metro line that ultimately would give the overall utilization level of the metro system; Finally, to analyze and evaluate the gathered results from various attributes in the simulation modeling software and identify the potential for prospective new services to be included into the existing metro system.

\subsection{Methodology}

This paper uses a systems approach: firstly, problem formulation accomplished through a deeper and comprehensive understanding of the current timetable. Secondly, 
developing a model using the SIMUL8 software that replicates the existing West Midlands Metro network accurately. Thirdly, evaluating the waiting time, working time, and utilization levels by running different simulations for several trials in a single day. Finally, the decisions can be made by analyzing and studying the obtained results and suggest the potential services that can be added up into the system. Also, this provides scope for future research work.

\subsection{Paper Organization}

The remainder of this manuscript is organized as follows: In Sect. 2, the characteristics of the West Midlands Metro are discussed. Section 3 illustrates the literature review, starting from the history of using timetables as center for evaluating the rail networks to the utilization of simulation software to replicate the system. The observations made from the timetable of the metro system are addressed in Sect. 4. Section 5 gives a brief introduction about SIMUL8 and explains the basic characteristics of the software, essential to carry out this study. Section 6 shows the system layout in SIMUL8. In Sect. 7 and Sect. 8, results from simulation model are presented, and the overall utilization levels of the metro system is generated. Section 9 reflects on the results obtained from the simulation model and analyzes the future context of the research work accompanied by references.

\section{Characteristics of West Midlands Metro}

West Midlands Metro is a tram line connecting the cities of Birmingham and Wolverhampton in the UK via the towns of West Bromwich and Wednesbury. The tram line connects Birmingham Grand Central and Wolverhampton St. George's. The light rail line operates only in one line and runs in the streets in urban areas, and opened conventional rail tracks link the towns and cities. The metro runs every 6-8 min during peak hours and runs a service every $15 \mathrm{~min}$ during nonpeak hours and Sundays all day. A total of 6.6 million people traveled by metro in the year 2016 [2]. There is an increase of $29 \%$ annual ridership observed. The line has 26 stations operating currently, and 9 more under construction. The line is $21 \mathrm{~km}$ (13 miles) long, using a $1435 \mathrm{~mm}$ standard gauge. The tramline is electrified by a $750 \mathrm{~V}$ DC over-head line. The West Midlands Metro has 21 "Urbos 3" vehicles for running the services, which can attain a top speed of $70 \mathrm{~km} / \mathrm{h}(43.5 \mathrm{mph})$. The original system started operating with a 16-tram fleet in 1999. The city center extension in Birmingham opened in May 2016, bringing the tram right into the city's heart along busy retail and commercial streets. This expansion was part of a $£ 128$-million plan that saw the purchase of a new " 21 - strong CAF Urbos 3" tram fleet, a renovated Wednesbury depot, and new stops for New Street station at St Chads, Bull Street, Corporation Street, and Grand Central. In Pinfold street, just around the corner from Grand Central, the line stops suddenly and attempts to logically stretch through Five Ways to Victoria and Centenary Squares and beyond to Edgbaston (Fig. 1).

Victoria Square is a significant architectural site, and catenary cables were not considered appropriate. Therefore, $840 \mathrm{~m}$ of twin track will have no overhead structure and the Urbos trams will run on battery power; the batteries are mounted in the roof, and a number of units have been fitted at the time of writing, and one unit now wears West Midlands Metro's new blue livery. There is another location where battery power is required-this time for more mundane structural clearance reasons. This is where, at the large Five Ways road junction, the tram uses the existing underpass. Work on other parts of the network is ongoing. The extension to Wolverhampton railway station from the former main line, currently being demolished and rebuilt, is due to be completed by 2020 . To the east, an application was made to build and operate the Birmingham Eastside Metro extension from Bull Street to Digbeth for a Transport and Works Act Order. The order would allow work to begin with the $1.7-\mathrm{km}$ (1.05-mile) extension that will serve Curzon Street's proposed HS2 station, providing connexions to New Street, Moor Street, and Snow Hill railway stations (Fig. 2).

The study is focused on the analysis of the timetable of the trams between Birmingham Grand Central and Wolverhampton St.George, using an event-based simulation.

\section{Literature Review}

Public timetable advises current and potential passengers about the services available, whereas the working timetable offers detailed information on a scheduled train operated by the train crew. The interested reader is referred to Schittenhelm [3], where timetables are classified into seven types based on type and purpose of service. According to the light rail system, the West Midlands Metro passenger service is subject to low schedule patterns, in which a metro system can have just two scheduled schedules during a day in service, e.g., daytime and evening + night time. That only means that the pattern needs to change twice. The minimum number is usually higher for long-haul rail traffic, since the number of trains is adjusted to the increasing travel demand during the day, more specifically. Timetables can be classified broadly into periodic and nonperiodic. The West Midlands Metro uses a periodic timetable which is structured. This 


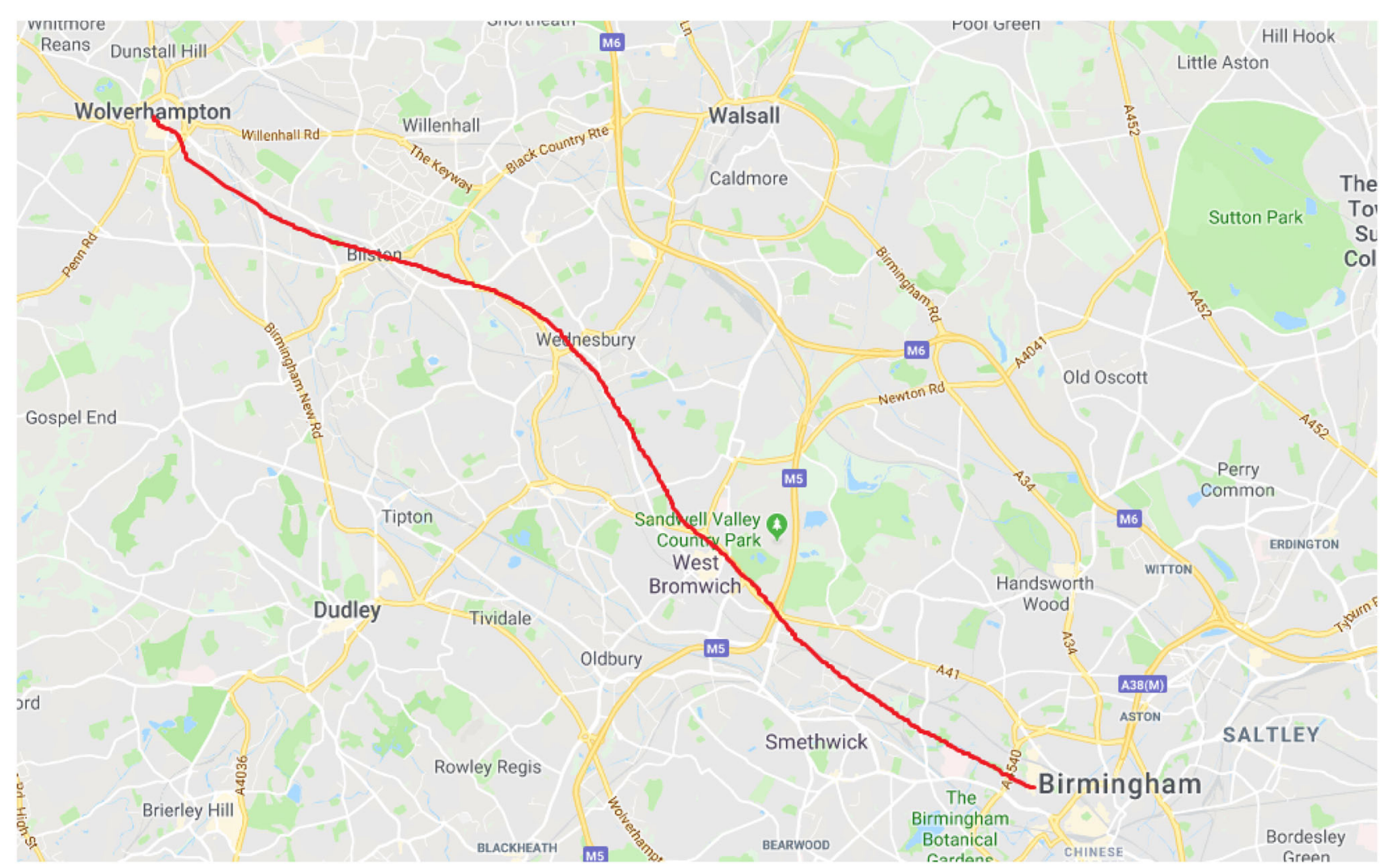

Fig. 1 Geographical overview of West Midlands Metro line

MIDLAND METRO

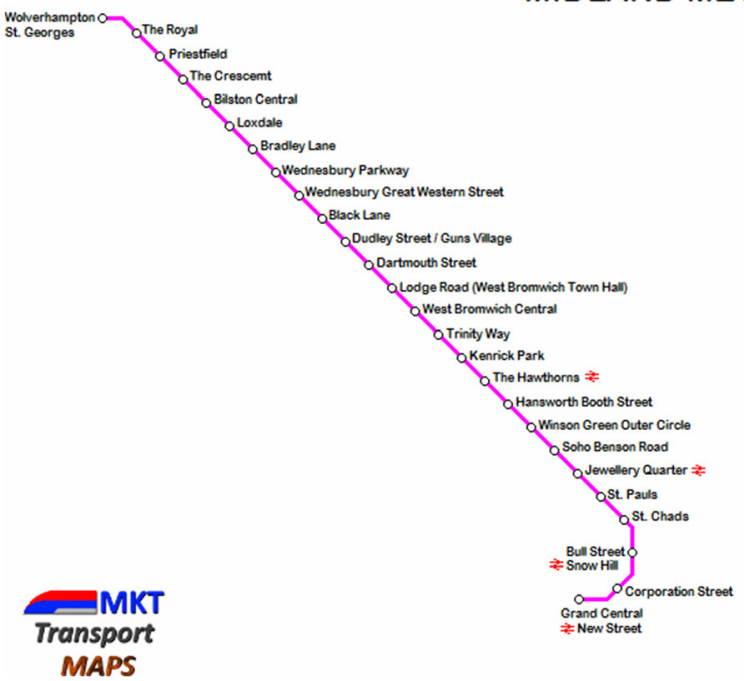

Fig. 2 The West Midlands Metro line

timetable shows the structure, since the trains run according to various schedules during the day. Nevertheless, there is no order in the nonperiodic cycle. It consists of separate scheduled routes based on demand for travel. Periodic schedules for the whole network are logical and consistent. They are a well-defined train network hierarchy that reduces the wait time for passengers arriving at stations on an alert basis.

The train timetable incorporates the rail network traffic rules and affects the rail traffic organization. Timetables involving moderate traffic and simple networks are easy to design using a computer. Nevertheless, manual and automatic support would be necessary for the design and execution of large networks with complex traffic. Analyzing timetable quality assesses the abilities of timetable administrators. It requires assessment of train track distribution and adjustment. The results of the schedule assessment indexes vary with infrastructure and traffic flux, making them unparalleled. There can be subjective inputs, such as expert scores, but this could lead to undesirable results, because experts have different views.

Two conventional ways to analyze and assess the performance of any system are usually analytical models and simulation methods. The analytical models provide a rapid understanding of how the system under consideration is performed and do not need to collect and process a significant amount of data [4]. Looking at a few instances of analytical methods being utilized to analyze the timetable, Feng $\mathbf{J}$ et al. proposed a data envelopment analysis (DEA) model to evaluate the quality of a timetable [5]. By taking the train paths as production units, a relative train path efficiency index was established. Each unit absorbs a 
certain amount of transport resources and generates feedback. The analysis was justified by checking with real life timetables and whether it reflects the even distribution of stops. However, this method could be useful only for analyzing the quality and corroborate with the existing system. It cannot help in giving any recommendations on the current timetables. Salido et al. proposed analytical and simulation techniques for assessing solidity in a full railway line [6]; they estimated the robustness of a timetable in their analytical method by means of the primary and secondary delay caused by a disruption. They also developed a disruption simulation method to test the robustness of a given schedule that incorporates unexpected events on a given schedule and conducts a rescheduling process and calculates the primary and secondary delays caused. Eventually, they suggested that the analytical model in single-line railway timetables would become an effective and useful option for light robustness evaluation.

Sels et al. built an analytical method that assesses the timetable for the total expected passenger time criterion, consisting in independently assessing the intervention in the timetable (ride, dwell, change, knock-on delay) in practice and stated that to be more efficient and justified by comparing two Belgian timetables using their application [7]. Goverede and Hensen stated that the art of creating such a schedule depends on several performance indicators related to individual train paths (running and dwelling), dependencies between train paths (headways, turning, transferring, etc.), and integrated train paths (corridors, networks) [8]. These metrics of performance include occupancy of infrastructure, stability of timetable, viability, durability, and adaptability. It is possible to evaluate the quality of the timetable design process by how these performance indicators are dealt with. They suggested a description of the performance measures, their timetable development significance, and interrelationships. Furthermore, four timetable levels have been advocated that serve the extent of timetable performance parameters taken into consideration during the timetable design.

But the empirical models are more restrictive than the models of simulation. Huang et al. used the discrete time simulation (DTS) to examine the efficiency of the railway network based on speed profile, and it was explained that Beijing Metro Line timetable is not solid enough [9]. Through using simulation, large dwell times in peak times are found to substantially increase system delay and energy consumption. Warg and Bohlin used the rail simulation to measure and calculate the predicted level of performance as a contribution to an economic assessment model to estimate the benefits of timetable changes, by adding a new method for integrating efficiency and economics by simulating the delay characteristics [10]. Solinen et al. stated that adapting to robustness in critical points (RCP) increases timetable robustness and presented the use of an approach for RCP optimization by generating an enhanced schedule which was evaluated using microscopic simulation and then assessed with several performance metrics [11]. The results indicate that, to accurately determine the impact of an improvement in RCP, it is important to use several key performance indicators (KPIs). The results also show, however, that the increase in RCP resulted in a decline in lateness and the risk of delay. Marinov et al. studied the train operating modes, scheduling, rail traffic control, dispatching problems, technical rail yard schemes, and performance of terminals. They presented an overview of analytical methods and suggested simulation methods that could be used to test these parameters [12].

Grube et al. presented an event-driven dynamic simulator for multiline metro systems in Chile's metro network and addressed its applicability to investigate different working strategies [13]. Mortaghi et al. created an eventbased simulation using ARENA to research the benefits of transporting urban freight by rail and demonstrate it as a feasible alternative to other common freight movement solutions on the market [14]. It is evident that the simulations have often been used in the analysis of railway systems, as they facilitate a more comprehensive analysis and obtain accurate representation of the actual systems [15]. They also give versatility to cover various scenarios and get an insight into different aspects.

SIMUL8 has been previously used to evaluate the rail system design and analyze delays and mitigation in the Tyne and Wear Metro [16]; using SIMUL8, a prototype of the Tyne and Wear metro was created and simulations were run from the primary source of information gathered with regards to the delays and early arrivals of trains. A set of mitigation strategies that helps in combating the delays and early arrivals in the system was recommended. Singhania analyzed the utilization rates of a railway service in the Glasgow metro using SIMUL8 [17]. They produced levels of utilization and concluded that additional freight trains can be incorporated into the network without disrupting existing timetables. This has been further justified, as it would improve the utilization levels of the system. Marinov and Viegas carried out a mesoscopic simulation modeling method using SIMUL8 to assess the movement of freight trains in the rail system [18]. A decomposition approach was used for simulation modeling purposes. This means dividing the discussed rail network into components such as rail lines, railway yards, railway stations, railway stations, and intersections. Marinov and Viegas evaluated the yard performances at tactical management level using a two steps approach [19]. They used analytical modeling with $\mathrm{G} / \mathrm{G} / \mathrm{m}$ queues and event-based simulation using SIMUL8. They reported that the simulations confirmed that the findings from the $\mathrm{G} / \mathrm{G} / \mathrm{m}$ queues had been verified. The 
simulations helped to study the yard performances in greater detail, such as behavior of yard personnel, which cannot be studied by G/G/m queues. They concluded that, with the increase in the number of freight trains, the utilization levels of the yard subsystem and yard personnel increased significantly. Potti et al. evaluated the utilization levels of a cross-city railway line in the United Kingdom using SIMUL8 and studied the possibility of moving urban freight by an existing railway line [20]. For this, they developed three possible scenarios that can move freight from Lichfield Trent Valley station to Birmingham New Street station, from where the freight can be distributed to the retail stores in Birmingham city or to the other parts of the UK by rail. They concluded that a scenario suggesting adding 108 freight trains to the actual system would have a significant impact on improving the lower utilization levels of the actual system and provide an efficient, economical, and sustainable method to move urban freight.

Woroniuk et al. studied and evaluated the performance of the rail route from Silla to Castellbisbal, in Eastern Spain [21]. They presented the utilization levels of the system in operation using ARENA simulation software and reported that the system is being underutilized. To enhance the utilization levels, they proposed different scenarios where the rail line could be used for carrying urban freight. They concluded that it would be possible to improve the utilization levels of the rail line. Marinov et al. determined whether the concept of urban freight rail is feasible or not through analyzing real cases where its practice had been already implemented [22]. They reported that the concept of urban freight rail is feasible by analyzing Newcastle Metro's potential to move urban freight by rail. Also, they provided solutions by developing a truck train model to carry urban freight.

For the purposes of this study, the evaluation of the metro timetable is implemented using SIMUL8, which is an event-based simulation modeling software founded based on a flow of units through a network. SIMUL8 was chosen for this work as it is evident from previous works that it would provide the flexibility required to carry out simulation trials incorporating different scenarios. The review of the literature indicates the robustness of SIMUL8 in researching and analyzing rail network.

\section{Observation from Current Timetable}

The observations gathered from analyzing the current timetable is key, as they provide inputs to most of the attributes in the simulation software. It is very important to understand the timetable, as it is the basic point of reference to the entire study. Timetable here determines several key attributes in simulation such as choosing the distribution for Start point attribute, designating the travel time between two stations, and the mean waiting time at each station. These three findings collected from timetable form the core of the input required to build the simulation model that replicates the actual system. These outputs determine the level of similarity of the model built to the existing real metro system. It is very important to build a model that appropriately describes the current metro system, as it provides accurate results about its utilization levels.

The Midlands Metro has trams at a frequency of every 6-8 min from Monday to Friday. Services run every 15 min during early mornings and evenings, including Sundays. The current metro system for passengers runs in a scheduled operating pattern. It can be observed from the timetable that the services start from the Wednesbury Parkway station every day (Fig. 3). The metro provides services from 05:00 am to 00:45 am daily for the passengers and takes $40 \mathrm{~min}$ to complete a trip from the start point to the end point in both the directions. The lines are operational for $20 \mathrm{~h}$ a day, and it is assumed that a period of $2 \mathrm{~h}$ during nonoperational hours is allotted for the construction and maintenance of the line daily. The average speed of the metro cars running in the line is calculated to be $50 \mathrm{~km} / \mathrm{h}$, approximately.

From this timetable, an observation on the number of metro cars originating from Birmingham Grand Central, Wednesbury Parkway, and Wolverhampton St. George's stations was developed. These observations are key, as they constitute one of the crucial inputs represented in the SIMUL8 software. For accurate representation in the software, the observations were split into four sections:

Section 1: Wednesbury Parkway to Birmingham Grand Central (Table 1).

Section 2: Birmingham Grand Central to Wolverhampton St. George's (Table 2)

Section 3: Wolverhampton St. George's to Birmingham Grand Central (Table 3).

Section 4: Wednesbury Parkway to Wolverhampton St. George's (Table 4).

There are 21 stations in this line, Wednesbury Parkway and Birmingham Grand Central being the entry and exit point, respectively. This section is significant, as it runs exclusively during nonpeak hours with a frequency of one service in every $15 \mathrm{~min}$. The travel time from Wednesbury Parkway to Birmingham Grand Central is $27 \mathrm{~min}$, and the round-trip time is $59 \mathrm{~min}$ (including $5 \mathrm{~min}$ of idle time at end point). The average waiting time at each station in this line, other than the terminal points, is taken as $0.8 \mathrm{~min}$ with reference to the timetable.

There are 26 stations in total in this line; this section represents the whole metro line, Birmingham Grand 


\section{MM1 Wolverhampton - Birmingham}

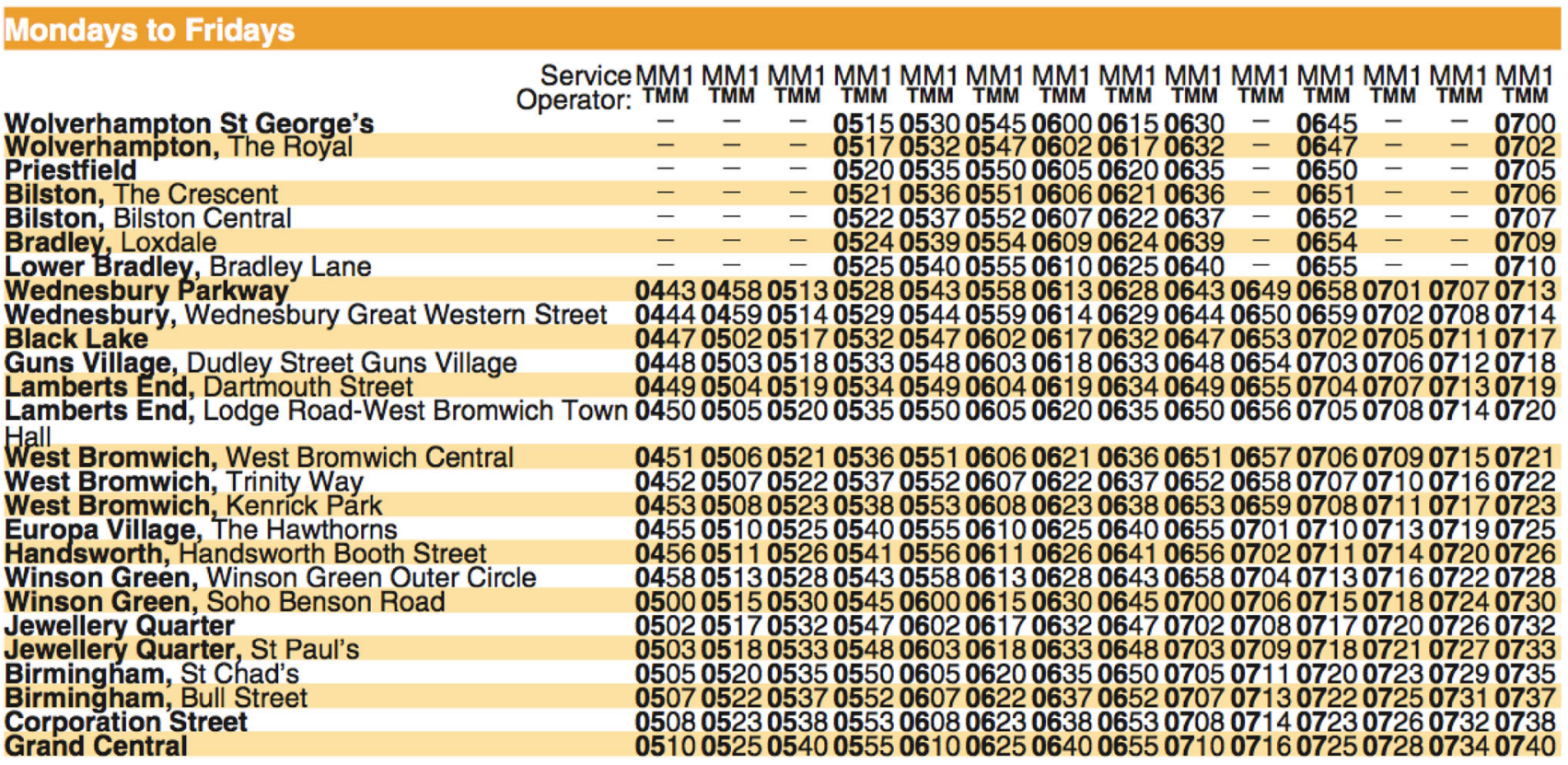

Fig. 3 Part of the timetable from Wolverhampton to Grand Central

Table 1 No. of services from Wednesbury Parkway to Birmingham Grand Central (section 1)

\begin{tabular}{lc}
\hline Time period & No. of trains \\
\hline Wednesbury Parkway to Birmingham Grand Central \\
04:45-06:45 & 4 \\
$06: 45-09: 20$ & 10 \\
$09: 20-16: 00$ & 7 \\
16:00-19:35 & 10 \\
19:35-20:13 & 7 \\
20:13-00:28 & 4 \\
\hline
\end{tabular}

Table 2 No. of services from Birmingham Grand Central to Wolverhampton St. George's (section 2)

\begin{tabular}{lc}
\hline Time period & No. of services \\
\hline Birmingham Grand Central to Wolverhampton St. George's \\
05:00-07:00 & 6 \\
07:00-09:00 & 15 \\
09:00-16:30 & 11 \\
16:30-19:00 & 15 \\
19:00-20:00 & 11 \\
20:00-00:15 & 6 \\
\hline
\end{tabular}

Central and Wolverhampton St. George's being the entry point and exit point, respectively. The trains run
Table 3 No. of services from Wolverhampton St. George's to Birmingham Grand Central (section 3)

\begin{tabular}{lc}
\hline Time period & No. of services \\
\hline Wolverhampton St. George's to Birmingham Grand Central \\
05:00-07:00 & 6 \\
07:00-09:00 & 15 \\
$09: 00-16: 30$ & 11 \\
16:30-19:00 & 15 \\
19:00-20:00 & 11 \\
20:00-00:15 & 6 \\
\hline
\end{tabular}

Table 4 No. of services from Wednesbury Parkway to Wolverhampton St. George's (section 4)

\begin{tabular}{ll}
\hline Time period & No. of services \\
\hline Wednesbury Parkway to Wolverhampton St. George's \\
04:57-06:42 & 2 \\
$06: 42-10: 00$ & 5 \\
10:00-16:16 & 4 \\
16:16-19:34 & 5 \\
19:34-20:00 & 4 \\
20:00-00:42 & 2 \\
\hline
\end{tabular}

continuously throughout the day with different frequencies depending on the peak hour and nonpeak hour. The travel 
time from Birmingham Grand Central to Wolverhampton St. George's is $40 \mathrm{~min}$, and the round-trip time is $85 \mathrm{~min}$ (including $5 \mathrm{~min}$ of idle time at end point). The average waiting time at each station in this line, other than the terminal points, is taken as $0.8 \mathrm{~min}$ with reference to the timetable.

There are 26 stations in total in this line; this section represents the whole metro line, Wolverhampton St. George's and Birmingham Grand Central being the entry point and exit point, respectively. The services run continuously throughout the day with different frequencies depending on the peak hour and nonpeak hour. The travel time from Wolverhampton St. George's to Birmingham Grand Central is $40 \mathrm{~min}$, and the round-trip time is $85 \mathrm{~min}$ (including $5 \mathrm{~min}$ of idle time at end point). The average waiting time at each station in this line, other than the terminal points, is taken as $0.8 \mathrm{~min}$ with reference to the timetable.

There are only 8 stations in this line, Wednesbury Parkway and Wolverhampton St. George's being the entry point and exit point of the line, respectively. This section is significant as it runs exclusively during nonpeak hours with a frequency of one service in every $15 \mathrm{~min}$. The travel time from Wednesbury Parkway to Wolverhampton St. George's is $13 \mathrm{~min}$, and the round-trip time is $31 \mathrm{~min}$ (including 5 min of idle time at end point). The average waiting time at each station in this line, other than the terminal points, is taken as $0.8 \mathrm{~min}$ with reference to the timetable.

The maximum number of trains running between two points in this line is 15 , and the minimum number of trains running between two points at any time is 2 . This huge difference can be observed as a result of variation in the number of trains in operation as per the peak hours and nonpeak hours.

This observation from timetable classifies the frequency of services running into three classes, depending on the time of the day they are running.

Peak hours: Services running during 07:00 am-09:00 am and 4:30 pm-7:00 pm are assumed to be peak hours, as the demand for the services and number of services running is highest in a day.

Off-peak hours: Services running from 09:00 am to 4:30 pm and from 7:00 pm to 8:00 pm are assumed to be off peak, as the number of services running in this phase are the second highest in a day.

Super off-peak hours: Services running from 05:00 am to $07: 00$ am and from 8:00 pm to 00:45 am are assumed to be super off-peak, as the number of services running in this phase is the lowest in a total day.

This segregation of a day into three different time zones is helpful further in computing the overall utilization levels of the West Midlands Metro line.

\section{Implementing in SIMUL8}

\subsection{Fundamentals of SIMUL8}

SIMUL8 is a computer simulation modelling package that can be used to build a simulation model that virtually represents a mechanism or a system. The aim is to imitate or emulate a real system, which helps exploring it, experimenting with it, and understanding it without altering the real system. SIMUL8 helps to compare different sets of scenarios and allows to formulate judgments after considering all angles. SIMUL8 demonstrates the workflow through a system, one incident at a time, with all the key interactions on the computer screen graphically displayed. Simulation is ideal for any situation involving a process flow. Because simulation is not dependent on any analytical formula, restrictive modeling assumptions do not limit simulation. However, simulation's biggest strength persists in its ability to correctly reflect the randomness seen in the actual world. Some of the areas that naturally encompass randomness include arrival patterns, service times, travel times in a network between stages, and many more. Therefore, the potential to design this diversity enables us to understand better how a system will work under a range of scenarios.

The whole simulation process in SIMUL8 revolves around processing work items. The basic components of the SIMUL8 environment include a system, work item, entrance, activity, queue, exit, resource, and route.

\subsection{Characteristics}

To evaluate the timetable of the metro line, this model must find the best possible movement of metro cars around the section of system by simulation and analysis. To achieve this, a simulation consisting of all the interactions between the metro cars and the network is important. The simulation research will reproduce all stations in the line. This requires creating an entry point as input for the metro cars into the system and an exit point to leave from the simulation. Briefly, this means that metro cars are supplied via a starting point into the system, and then, a sequence of operations that mimic stations, queues, simulate lines, and then exit the system by an end point. The system does not interfere with travel flows in the clockwise and anticlockwise direction. This makes metro cars unable to move on opposing lines, which in turn, avoids any possible blockages, as each platform has to be used only for a single travel direction. 


\subsection{Activities}

'Activity' refers to a place where work on work items takes place. There will be many standard resources used when carrying out an activity. In this case, the activity module makes it possible to model the stations where the train cars stop in the system. It is limited by permitting only one metro car at a time on one platform (activity). The work center is the waiting time for each operation given by the feedback of the average waiting time from the analysis at each platform. There are two activities established between any two stations for the purposes of this research. Each activity reflects the time of service at each station, i.e., the time interval between the arrival and departure of the subway cars. The other activity describes the time of travel between any two stations.

\subsection{Queues}

The "queues" model cumulation of entities. They usually precede the activities module for which the stacked entities wait because of lack of resources. In this case, module 'queue' is used to restrict the track capacity between any two stations. Queues are limited to have a maximum capacity of one, which means that only one metro car is accessible in each segment of line at a time. This satisfies the headway allotment in the metro network, as it ensures that there is always a certain width between adjacent train cars to avoid accidents and interrupt the services.

\subsection{Start Point and End Modules}

The train cars entering the system are created by the objects 'start point'. The metro cars' arrival times can be set to a random or fixed timetable. For the purpose of this study, start points will be configured to suit a regular schedule and follow similar arrival trends as prescribed in the timetable of the metro for that period of study. There is one metro service for every 6 min running from Birmingham Grand Central to Wolverhampton St. George's during the peak hours, which is considered for the purpose of this study.

The implication of metro cars going out of the system is facilitated by using 'end' modules. These end modules are present at the end of every section considered. For this study, four sections are considerer; For example, consider Section 2, i.e., Birmingham Grand Central-Wolverhampton St. George's. If we consider this as a clockwise loop, here the end point of the clockwise loop will be Wolverhampton St. George's. A metro car fulfills its clockwise cycle and waits at St. George's before going back to the anticlockwise loop, which is like a metro car being disposed of and reentering.

\section{System Layout and Implementation of the Model}

'Utilization levels', in this study, refers to the actual time the line is under operation. It is derived by computing the operating times for all the stations existing in the metro system. The operating times and waiting times for all the stations in both the directions of travel are generated by implementing the metro system in the SIMUL8 simulation software, which is thoroughly explained in the following sections of this paper. This method of computing utilization levels by recreating the entire West Midlands Metro system in SIMUL8 software presents the opportunity to generate the accurate operating times of all the stations and the queue lines leading to a station, from which the overall utilization levels can be generated. The literature review also presents the substantiate evidence of the analysis achieved by using SIMUL8 software.

As the West Midlands Metro line consists of 26 stations in a single line, the layout has been made using start point, queue, activity, and end point modules. Connecting these attributes replicates the exact metro system. The whole metro line has been segregated into four sections for the purpose of a detailed study, as mentioned in Sect. 5. Hence, four different models have been created to run the simulations, and all the four sections utilizations have been analyzed and presented. Figure 4 shows the model for Section 1, i.e., Birmingham Grand Central to Wolverhampton St. George's. At the start point, the number of metro cars coming into the system in the specified interval is derived from the West Midlands Metro timetable. The data of the incoming metro cars into the system was extracted from the spread sheet which resembles the original timetable. All the other parameters such as service time at each station or mean travel time between two stations were derived from the timetable. Similarly, four other models were created, and the simulations were run for a 1-day period to collect the average waiting time and average working time that would provide the utilization level of each activity center, i.e., each station along the metro line.

Simulation modeling is crucial, as it enables to study the models and systems that are too complicated for analytical or numerical treatment. It provides detailed relations that an analytical or a mathematical model cannot produce or visualize. In this context, using the timetable as a reference incorporates all the KPIs such as speed profiles, acceleration profiles, dwell time or travel time during the simulated period. Incorporating timetable as reference for the purpose of this study provides key inputs for attributes such as start point, end point and frequency at which the metro cars are fed into system. 


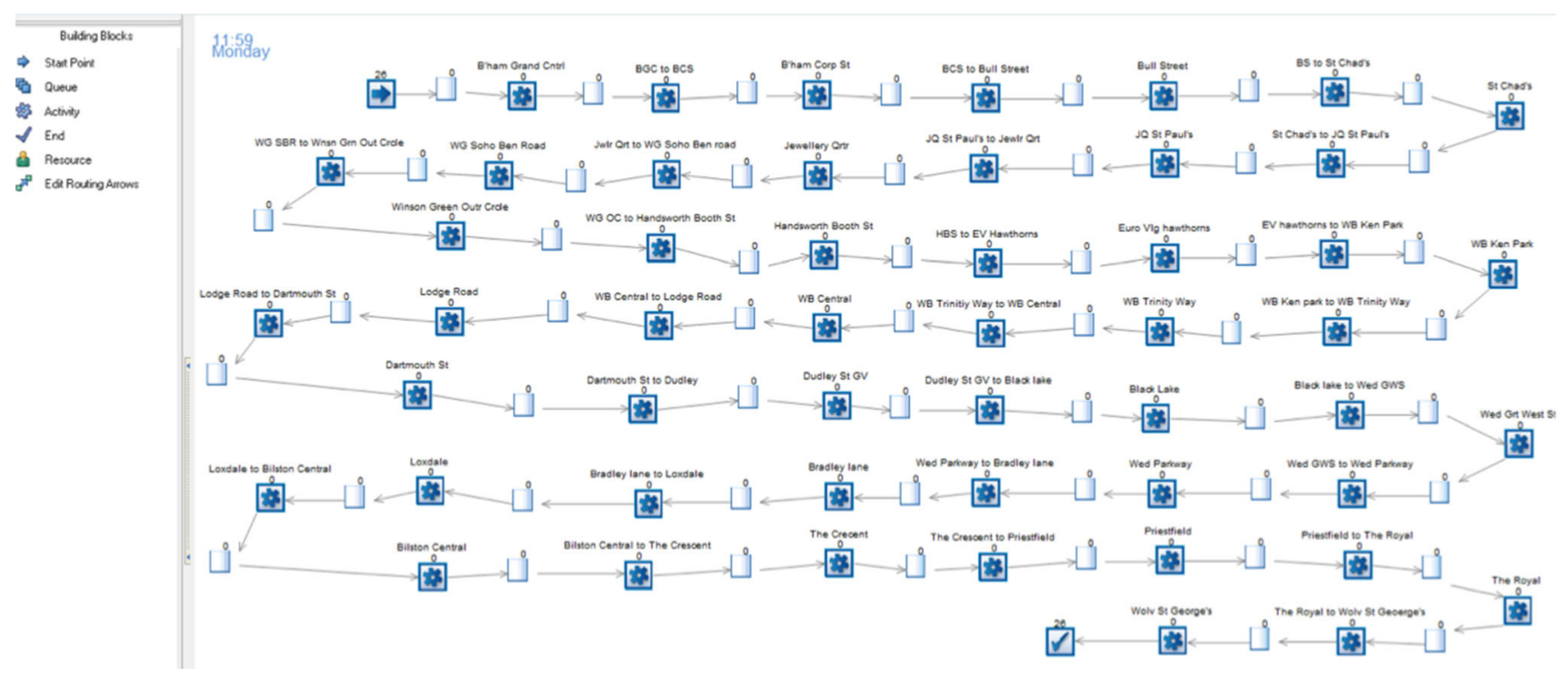

Fig. 4 Simulation layout of Section 1

\section{Generation of Utilization Levels}

To achieve the most accurate utilization levels of the metro line, the simulation process is divided into two sections. The utilization levels are computed separately for each section and presented together as a whole for the system at the end of this study. The two sections that represent the line are:

Clockwise direction: Birmingham Grand Central to Wolverhampton St. George's

Anti-clockwise direction: Wolverhampton St. George's to Birmingham Grand Central

It is important to obtain and study the simulation results of a single line in two different directions, as they have different timetables for different directions. This creates two sets of different operating times and waiting times for a single station in the line, which cumulated together gives the overall operating and waiting time of a single station, from which the overall utilization level of the system is evaluated.

\subsection{Section 1: Clockwise Direction}

\subsubsection{Birmingham Grand Central to Wolverhampton St. George's}

In order to compute the utilization levels of the system while considering the clockwise direction, it is needed to generate the operating times and waiting times of all the stations in the metro system. The total operating times are represented as a sum of three sections namely peak hours, off-peak hours, and super off-peak hours, which were derived from an assumption made at the end of Sect. 4. Hence, the results are also presented as per the three travel times. The simulation was carried out differently for different travel times. However, the layout remains same for each case, as only the frequency and time at which metro cars are fed into the system varied. Collecting and analyzing results as per different travel times provide a wider field of results that would be useful to identifying gaps in the system by providing a more robust analysis.

7.1.1.1 Utilization Levels During Peak Hours As mentioned in the earlier section, peak hours refer to metro cars operating between 07:00 am to 09:30 am and 4:00 pm to 7:00 pm. Table 5 presents the detailed information about the utilization levels of the metro cars running during peak hours in the clockwise direction, i.e., from Birmingham Grand Central to Wolverhampton St. George's. The metro cars in this part of the day run on a frequency of one service for every $6 \mathrm{~min}$. The start point for this section is Birmingham Grand Central, from where the metro cars are introduced into the system in the simulation software. The times at which the metro cars are added into the system is inferred from the timetable.

This part of the line was simulated for a time period of 05:30 am that refers to the results collection period in the SIMUL8 software, and five trails were run in the simulation process. It is observed that the terminal stations, i.e., Birmingham Grand Central and Wolverhampton St. George's, are operating $67.50 \%$ of the time, while the other stations in the line are only being operated for $10.80 \%$ of the time. The terminal stations are idle for $32.50 \%$ of the 
Table 5 Operating and waiting times of the system during peak hours in clockwise direction

\begin{tabular}{lcccc}
\hline Station & Operating time (min) & Waiting time (min) & Operating \% & Waiting \% \\
\hline Birmingham Grand Central & 213.98 & 103.03 & 67.50 & 32.50 \\
Other stations in the line & 34.24 & 282.76 & 10.80 & 89.20 \\
Wolverhampton St. George's & 213.98 & 103.03 & 67.50 & 32.50 \\
Total utilization \% & $15.16 \%$ & & \\
\hline
\end{tabular}

Table 6 Operating and waiting times of the system during off-peak hours in clockwise direction

\begin{tabular}{lllll}
\hline Station & Operating time (min) & Waiting time (min) & Operating \% & Waiting \% \\
\hline Birmingham Grand Central & 303.53 & 166.47 & 64.58 & 35.42 \\
Other stations in the line & 48.55 & 421.45 & 10.33 & 89.67 \\
Wolverhampton St. George's & 303.53 & 166.47 & 64.58 & 35.42 \\
Total utilization \% & $14.05 \%$ & & \\
\hline
\end{tabular}

time, whereas the other stations in the line are idle for $89.20 \%$ of the time. This accounts for total utilization of $15.16 \%$ of the time during peak hours in clockwise direction.

7.1.1.2 Utilization Levels during Off-Peak Hours Offpeak hours refer to metro cars operating during 09:00 am4:00 pm and 7:00 pm-8:00 pm. Table 6 presents the detailed information about the utilization levels of the metro cars running during off-peak hours in the clockwise direction, i.e., from Birmingham Grand Central to Wolverhampton St. George's. The metro cars in this part of the day run on a frequency of one service for every $8 \mathrm{~min}$. The start point for this section is Birmingham Grand Central, from where the metro cars are introduced into the system in the simulation software. The times at which the metro cars are added into the system are inferred from the timetable.

This part of the line was simulated for a time period of 08:00 am, and 5 trails were run in the simulation process. It is observed that the terminal stations, i.e., Birmingham Grand Central and Wolverhampton St. George's are operating $64.58 \%$ of the time, while the other stations in the line are only being operated for $10.33 \%$ of the time. The terminal stations are idle for $35.42 \%$ of the time, whereas the other stations in the line are idle for $89.67 \%$ of the time. This accounts for total utilization of $14.05 \%$ of the time during off-peak hours in clockwise direction.

\subsubsection{Utilization Levels during Super Off-Peak}

Hours Super off-peak hours refer to metro cars operating during 05:00 am-07:00 am and 8:00 pm-00:45 am. Table 7 presents the detailed information about the utilization levels of the metro cars running during off-peak hours in the clockwise direction, i.e., from Birmingham
Grand Central to Wolverhampton St. George's. The metro cars in this part of the day run on a frequency of one service for every $15 \mathrm{~min}$. The start point for this section is Birmingham Grand Central, from where the metro cars are introduced into the system in the simulation software. The times at which the metro cars are added into the system are inferred from the timetable.

This part of the line was simulated for a time period of 06:40 am, and five trails were run in the simulation process. It is observed that the terminal stations, i.e., Birmingham Grand Central and Wolverhampton St. George's, are operating $30.77 \%$ of the time, while the other stations in the line are only being operated for $4.92 \%$ of the time. The terminal stations are idle for $69.23 \%$ of the time, whereas the other stations in the line are idle for $95.08 \%$ of the time. This accounts for total utilization of $6.9 \%$ of the time during super off-peak hours in clockwise direction.

\subsubsection{Total Utilization in Clockwise Direction}

Table 8 presents the total operating times, waiting times, and utilization levels of the system when run in clockwise direction, i.e., Birmingham Grand Central to Wolverhampton St. George's. These attributes are derived from the travel time wise utilization levels presented earlier in this section. Terminal stations, i.e., Birmingham Grand Central and Wolverhampton St. George's are observed to be operating $44.27 \%$ of the time, while the other stations in the line are each being operated for $7.08 \%$ of the total time in a day. The total utilization level of the metro line in clockwise direction from Birmingham Grand Central to Wolverhampton St. George's is reported to be $9.94 \%$.

The total utilization levels of the system are generated using the below mentioned formula. 
Table 7 Operating and waiting times of the system during super off-peak hours in clockwise direction

\begin{tabular}{lllrr}
\hline Station & Operating time (min) & Waiting time (min) & Operating \% & Waiting \% \\
\hline Birmingham Grand Central & 120 & 270 & 30.77 & 69.23 \\
Other stations in the line & 19.19 & 370.81 & 4.92 & 95.08 \\
Wolverhampton St. George's & 120 & 270 & 30.77 & 69.23 \\
Total utilization \% & $6.9 \%$ & & \\
\hline
\end{tabular}

Table 8 Total utilization of the metro line in clockwise direction

\begin{tabular}{|c|c|c|c|c|}
\hline Station & Operating time $(\mathrm{min})$ & Waiting time (min) & Operating \% & Waiting \% \\
\hline Birmingham Grand Central & 637.50 & 539.50 & 44.27 & 37.46 \\
\hline Other stations in the line & 101.98 & 1075.03 & 7.08 & 74.65 \\
\hline Wolverhampton St. George's & 647.50 & 539.50 & 44.27 & 37.46 \\
\hline Total utilization $\%$ & $9.94 \%$ & & & \\
\hline
\end{tabular}

Total utilization $\%$

$$
\begin{aligned}
& =\left(\frac{\text { Sum of operating times of all the stations in the metro line }}{\text { Total number of minutes in a day }}\right) \\
& \times 100
\end{aligned}
$$

\subsection{Section 2: Anticlockwise Direction}

\subsubsection{Wolverhampton St. George's to Birmingham Grand Central}

7.2.1.1 Utilization Levels during Peak Hours Peak hours refer to the travel times of metro cars during 07:00 am09:00 am and 4:30 pm-7:00 pm. Table 9 presents the detailed information about the utilization levels of the metro cars running during peak hours in the anticlockwise direction, i.e., from Wolverhampton St. George's to Birmingham Grand Central. The metro cars in this part of the day run on a frequency of one service for every $6 \mathrm{~min}$. The start point for this section is Wolverhampton St. George's, from where the metro cars are introduced into the system in the simulation software. The times at which the metro cars are added into the system are inferred from the timetable.
This part of the line was simulated for a time period of 05:00 am, and five trails were run in the simulation process. It is observed that the terminal stations, i.e., Wolverhampton St. George's and Birmingham Grand Central, are operating $65.28 \%$ of the time, while the other stations in the line are only being operated for $10.44 \%$ of the time. The terminal stations are idle for $34.72 \%$ of the time, whereas the other stations in the line are idle for $89.56 \%$ of the time. This accounts for total utilization of $14.65 \%$ of the time during peak hours in anticlockwise direction.

7.2.1.2 Utilization Levels during Off-Peak Hours As mentioned above, off-peak hours refer to the metro cars running during 09:00 am-4:30 pm and 7:00 pm-8:00 pm. Table 9 presents the detailed information about the utilization levels of the metro cars running during off-peak hours in the anticlockwise direction, i.e., from Wolverhampton St. George's to Birmingham Grand Central. The metro cars in this part of the day run on a frequency of one service for every $8 \mathrm{~min}$. The start point for this section is Wolverhampton St. George's, from where the metro cars are introduced into the system in the simulation software. The times at which the metro cars are added into the system are inferred from the timetable (Table 10).

Table 9 Operating and waiting times of the rail system during peak hours in anticlockwise direction

\begin{tabular}{lllll}
\hline Station & Operating time (min) & Waiting time (min) & Operating \% & Waiting \% \\
\hline Birmingham Grand Central & 195.84 & 104.16 & 65.28 & 34.72 \\
Other stations in the line & 31.32 & 268.8 & 10.44 & 69.56 \\
Wolverhampton St. George's & 195.84 & 104.16 & 65.28 \\
Total utilization \% & $14.65 \%$ & & \\
\hline
\end{tabular}


Table 10 Operating and waiting times of the rail system during off-peak hours in anticlockwise direction

\begin{tabular}{lllll}
\hline Station & Operating time (min) & Waiting time (min) & Operating \% & Waiting \% \\
\hline Birmingham Grand Central & 320 & 180 & 64 & 36 \\
Other stations in the line & 51.20 & 448.80 & 10.24 & 89.76 \\
Wolverhampton St. George's & 320 & 180 & 64 \\
Total utilization \% & $14.37 \%$ & & 36 \\
\hline
\end{tabular}

This part of the line was simulated for a time period of 08:00 am, and five trails were run in the simulation process. It is observed that the terminal stations, i.e., Wolverhampton St. George's and Birmingham Grand Central, are operating $64 \%$ of the time, while the other stations in the line are only being operated for $10.24 \%$ of the time. The terminal stations are idle for $36 \%$ of the time, whereas the other stations in the line are idle for $89.76 \%$ of the time. This accounts for total utilization of $14.37 \%$ of the time during off-peak hours in anticlockwise direction.

\subsubsection{Utilization Levels during Super Off-Peak}

Hours Super off-peak hours refer to metro cars operating during 05:00 am-07:00 am and 8:00 pm-00:45 am. Table 11 presents the detailed information about the utilization levels of the metro cars running during super offpeak hours in the anticlockwise direction, i.e., from Wolverhampton St. George's to Birmingham Grand Central. The metro cars in this part of the day run on a frequency of one service for every $15 \mathrm{~min}$. The start point for this section is Wolverhampton St. George's, from where the metro cars are introduced into the system in the simulation software. The times at which the metro cars are added into the system are inferred from the timetable.

This part of the line was simulated for a time period of 06:00 am, and five trails were run in the simulation process. It is observed that the terminal stations, i.e., Wolverhampton St. George's and Birmingham Grand Central, are operating $32.81 \%$ of the time, while the other stations in the line are only being operated for $5.25 \%$ of the time. The terminal stations are idle for $67.19 \%$ of the time, whereas the other stations in the line are idle for $94.75 \%$ of the time. This accounts for total utilization of $7.37 \%$ of the time during super off-peak hours in anticlockwise direction.

\subsubsection{Total Utilization in Clockwise Direction}

Table 12 presents the total operating times, waiting times and utilization levels of the system when running in clockwise direction, i.e., Birmingham Grand Central to Wolverhampton St. George's. These attributes are derived from the travel time wise utilization levels presented above in this section.

Terminal stations, i.e., Wolverhampton St. George's and Birmingham Grand Central, are observed to be operating $43.11 \%$ of the time, while the other stations in the line are each being operated for $6.90 \%$ of the total time in a day. The total utilization level of the metro line in clockwise direction from Birmingham grand Central to Wolverhampton St. George's is reported to be $9.68 \%$.

\section{Results}

The operating times and idle times of all the 26 stations in the line during different travel times were collected using SIMUL8 software. This helps in determining the overall utilization level of the entire West Midlands Metro system. Table 13 presents the detailed information about the overall operating, waiting times, and percentage of times of all the stations in different directions and different travel times. Given the need of most organizations running the metro services, a 2-h maintenance period was allocated for the line, during which no services operate. The use of the line is considered to be $100 \%$ during this time, as the maintenance work carried out is assumed to be required and useful.

The overall utilization level in a single day for West Midlands Metro line is computed to be $24.6 \%$. The

Table 11 Operating and waiting times of the system during super off-peak hours in anticlockwise direction

\begin{tabular}{lclrr}
\hline Station & Operating time (min) & Waiting time (min) & Operating \% & Waiting \% \\
\hline Birmingham Grand Central & 104.99 & 215.1 & 32.81 & 67.19 \\
Other stations in the line & 16.80 & 303.20 & 5.25 & 94.75 \\
Wolverhampton St. George's & 104.99 & 215.1 & 32.81 & 67.19 \\
Total utilization \% & $7.37 \%$ & & \\
\hline
\end{tabular}


Table 12 Total utilization of the metro line in clockwise direction

\begin{tabular}{|c|c|c|c|c|}
\hline Station & Operating time (min) & Waiting time (min) & Operating \% & Waiting \% \\
\hline Wolverhampton St. George's & 620.83 & 499.17 & 43.11 & 34.66 \\
\hline Other stations in the line & 99.32 & 1020.68 & 6.90 & 70.88 \\
\hline Birmingham Grand Central & 620.83 & 499.17 & 43.11 & 34.66 \\
\hline Total utilization \% & $9.68 \%$ & & & \\
\hline
\end{tabular}

Table 13 Overall utilization levels of the metro line

\begin{tabular}{llccc}
\hline Station & Operating time (min) & Waiting time (min) & Operating \% & Waiting \% \\
\hline Birmingham Grand Central & 749.17 & 690.83 & 52.03 & 47.97 \\
Other stations in the line & 321.30 & 1118.71 & 22.31 & 77.69 \\
Wolverhampton St. George's & 749.17 & 690.83 & 52.03 & 47.97 \\
Overall utilization \% & $24.6 \%$ & &
\end{tabular}

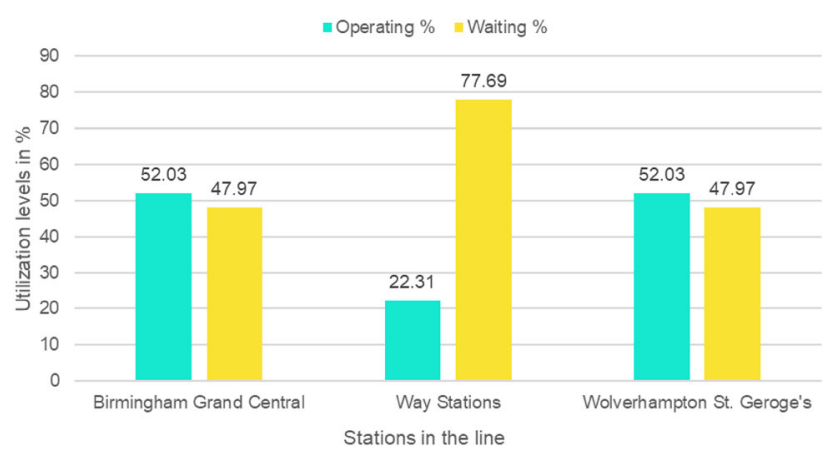

Fig. 5 Station wise utilization levels

utilization rates of the 24 stations between the terminal stations are identical at $22.31 \%$, while the terminal stations are reported to be utilized for $52.03 \%$ of the time.

The operating times for the terminal stations are obtained by taking the average of the total operating times of terminal stations in each direction. The average time was taken instead of the total time because the terminal stations are equipped with the track towards only one direction, unlike the way stations that have tracks and platforms for both directions, namely clockwise and anticlockwise. The total utilization levels of the system are computed from the below formula

Total Utilization levels $\%$

$$
=\left(\frac{\text { Sum of operating times of all stations in the line }}{\text { Total duration of the day in minutes }}\right) \times 100
$$

Figure 5 shows the station wise utilization levels for the West Midlands Metro. The observed higher utilization levels at terminal stations is due to the longer dwell times, as the metro car changes its direction of travel at terminal stations. This usually requires longer times.

\section{Concluding Remarks and Future Scope of Research}

The existing timetable of the West Midlands Metro was evaluated, and the utilization rates of the system were studied by developing an event-based simulation model. Using the timetable as a reference to several attributes in the simulation model, the metro model was implemented in the SIMUL8 simulation computer software.

The West Midlands Metro system was analyzed in both the clockwise and anticlockwise directions separately for different times of the day namely peak, off-peak, and super off-peak. This segregation was done based on the frequency of metro cars running in the line. The results report that the overall utilization of the metro system is $24.6 \%$, and it is considered that the system is being underutilized. However, the contribution of the terminal stations, i.e., Birmingham Grand Central and Wolverhampton St. George's, performance is significantly higher when compared with the other way stations. The lower utilization levels during off-peak and super-off peak reflect the gap in the system.

This situation is ideal to find ways to boost the utilization rates of the entire system. Few additional services included into the system possess a higher chance for improved utilization levels, however it would be of interest to understand and explore the demand for additional services carrying passengers. It calls for the requirement to introduce new marketing strategies to be employed in order to increase the ridership that in turn improves the utilization levels of the system. Extending the existing metro line to furthermore regions and including several other metro stations into the line can help to improve the performance of the system, which is currently being carried out. 
Services like moving urban freight by the West Midlands Metro system can also be explored, as the region is considered to have a high value demand for logistics operations related to urban freight and addresses key concerns of achieving energy efficiency and reducing pollution.

The existing timetable for the West Midlands Metro was analyzed to assess the performance of the system. It would be beneficial to research further any disruptions caused in the timetable and analyze potential delays as a result of such disruptions. Delay mitigating strategies could be devised to improve the quality of the West Midlands Metro system overall.

More importantly, there is scope for further work that analyzes the introduction of new services, such as using the existing system for moving urban freight. Such services would be very beneficial as they would generate new businesses, improve present level of utilization, and contribute to the region's sustainable urban logistics mode that decreases congestion, emissions, and traffic. There is also the potential to use the current metro line to transport and dispose of non-time-dependent commodities such as trash from companies situated along the metro line at recycling centers. In addition to this investigation, it would also be worth studying the modifications that can be made to the existing system such that it would support for the urban freight movement. It would be of importance to study if there is demand for such services in the region before implementing it. In this regard, market research aimed at identifying potential investors to transfer urban freight by metro in this region would be beneficial.

Open Access This article is distributed under the terms of the Creative Commons Attribution 4.0 International License (http://crea tivecommons.org/licenses/by/4.0/), which permits unrestricted use, distribution, and reproduction in any medium, provided you give appropriate credit to the original author(s) and the source, provide a link to the Creative Commons license, and indicate if changes were made.

\section{References}

1. West Midlands Combined Authority (2017) https://www.wmca. org.uk/news/national-survey-sees-satisfaction-soar-on-midlandmetro-as-passenger-numbers-near- $8 \mathrm{~m} /$. Accessed 12 June 19

2. Transport for West Midlands, Midland Metro passenger numbers set to top seven million (2017) https://www.tfwm.org.uk/news/ midland-metro-passenger-numbers-set-to-top-seven-million/. Accessed 14 June 19

3. Schittenhelm BH (2014) Quantitative methods for assessment of railway timetables. Ph.D. thesis, DTU, annual report year. http:// orbit.dtu.dk/en/publications/quantitative-methods-for-assess ment-of-railway-timetables(0407117c-d4d0-43b8-a95dda5991ef6dff).html. Accessed 10 May 19

4. Marinov M, Mortimer P, Zunder T, Islam D (2011) A steady state analysis for yard performances. J Transp Lit 5(1):33-49

5. Feng J, Yu D, Ni SQ (2017) An objective train timetabling quality evaluation method. Math Probl Eng 2017, 3047963

6. Salido MA, Barber F, Ingolotti L (2012) Robustness for a single railway line: analytical and simulation methods. Expert Syst Appl 39:13305-13327

7. Sels P, Cattrysse D, Vansteenwegen P (2015) Practical macroscopic evaluation and comparison of railway timetables. Transp Res Procedia 10:625-633

8. Goverede RMP, Hensen IA (2013) Performance indicators for railway timetables. In: IEEE ICIRT

9. Huang H, Li K, Wang Y (2018) A simulation method for analysing and evaluating rail system performance based on speed profile. Syst Sci Syst Eng 27(6):810-834

10. Warg J, Bohlin M (2016) The use of railway simulation as an input to economic assessment of timetables. J Rail Transp Plan Manag 6(3):225-270

11. Solinen E, Nicholson G, Peterson A (2017) A microscopic evaluation of railway timetable robustness and critical points. J Rail Transp Plan Manag 7:207-223

12. Marinov M, Giovanni LD, Bellisai G, Clevermann J, Mastellou A, Victoria D, Deleva L (2012) Analysis of rail yard and terminal performances. J Transp Lit 8:2

13. Grube P, Nunez F, Cipriano A (2010) An event driven simulator multiline metro system and its application to Santiago de Chile metropolitan rail network. Simul Model Pract Theory 19(1):393-405

14. Mortaghi A, Marinov M (2012) Analysis of urban freight by rail using event-based simulation. Simul Model Pract Theory 25:73-89

15. Tan W, Chai Y, Liu Y (2011) A message-driving formalism for modeling and simulation of multi-agent supply chain systems. J Syst Sci Syst Eng 20(4):385-399

16. Wales J, Marinov M (2015) Analysis of delays and delay mitigation on a metropolitan railway network using event-based simulation. Simul Model Pract Theory 52:55-57

17. Singhania V, Marinov M (2016) An event-based simulation model for analysing the utilization levels of a railway line in urban area. PROMET 29(5):521-528

18. Marinov M, Viegas J (2011) A mesoscopic simulation modelling methodology for analysing and evaluating freight train operations in a rail network. Simul Model Pract Theory 19:516-539

19. Marinov M, Viegas JM (2012) Tactical management of rail freight transportation services: evaluation of yard performance. Transp Plan Technol 34(4):363-387

20. Potti P, Marinov M, Sweeney E (2019) A simulation study on the potential of moving urban freight by a cross-city railway line. Sustainability 11(21):6088. https://doi.org/10.3390/su11216088

21. Woroniuk C, Marinov M (2012) Simulation modelling to analyze the current level of utilization of sections along a rail route. J Transp Lit 7(2):235-252

22. Marinov M, Giubilei F, Gerhardt M, Ozkan T, Stergiou E, Papadopol M, Cabecinha L (2013) Urban freight movement by rail. J Transp Lit 7(3):87-116 